\title{
The Frontier of Biobased Polymers: Synthesis, Characterization, Application, and Sustainability Assessment
}

\author{
Daniela Saviello, ${ }^{1}$ Daniele Cespi, ${ }^{2}$ Vinay Sharma, ${ }^{3}$ Shida Miao, ${ }^{4}$ and Raffaele Cucciniello ${ }^{5}$ \\ ${ }^{1}$ Tyndall National Institute, University College Cork, Dyke Parade, Cork, Ireland \\ ${ }^{2}$ Environmental Management and Consulting (EMC) Innovation Lab S.r.l., Viale Italia 29, 47921 Rimini, Italy \\ ${ }^{3}$ Apollo Tyres Ltd., Chennai, India \\ ${ }^{4}$ School of Engineering and Applied Science, The George Washington University, Washington, DC 20052, USA \\ ${ }^{5}$ Dipartimento di Chimica e Biologia “Adolfo Zambelli”, Università di Salerno, Via Giovanni Paolo II, 132-84084 Fisciano, Italy
}

Correspondence should be addressed to Raffaele Cucciniello; rcucciniello@unisa.it

Received 29 August 2017; Accepted 29 August 2017; Published 24 October 2017

Copyright (C) 2017 Daniela Saviello et al. This is an open access article distributed under the Creative Commons Attribution License, which permits unrestricted use, distribution, and reproduction in any medium, provided the original work is properly cited.

In the last years, research perspectives in the field of biobased polymers have attracted increasing interest both in industrial and in academic environments forward to a future based on renewable resources. Relevant papers and exhaustive reviews have been published concerning synthesis, extraction, and preparation of biobased monomers and characterization and applications of new biobased polymers.

The importance of biobased feedstocks in the chemical industry is continuously growing and their use is predicted to surpass that of products obtained by nonrenewable fossil sources in the near future.

As a matter of fact, recent statistics have shown that the production of biobased polymers is not intended to recess, but it rather will triple those that the manufacture achieved in 2013. Predictions for 2020 expect production of over 17 million tons. The market in Asia is expected to explode, taking the lion's share of capital investment, while in Europe the bioplastics market will be over 6.7 Mt $(+400 \%$ compared to 2013) by 2018 . More than $74 \%$ of the production (5 Mt) will be represented by bio-polyethylene terephthalate (bio-PET). Figure 1 describes the EU breakdown. As stated, bio-PET is dominant ( $+37 \%$ compared to the 2013 value). The other interesting portion is the green one, representing biodegradable polymers: around $17 \%$ in 2018 (PLA and polyesters constitute the majority).

The substitution of fossil-based resources with molecules extracted from biomass represents one of the main goals of Green Chemistry (see 7th principle). The importance behind this replacement is associated with two main reasons: the usage of renewable sources rather than limited ones and the development of a low-carbon economy, reducing wastes and minimizing the amount of resources. For example, the replacement of $20 \%$ of the carbon content related to the whole PET current production (around 37.5 billion tons) with biobased sources could contribute to the reduction of $\mathrm{CO}_{2}$ emission by 17 million tons. Moreover, another reason should be also taken into consideration: biobased industry will drive the economy by creating new opportunities and jobs. In general, the environmental sustainability of these biobased plastics is usually related to the embodied loads of the building block monomers used in the manufacture.

In this special issue, there are original research articles that uncover the development of new biobased polymers, including their synthesis, processing, characterization, and applications. The topics in this special issue specially include the following: (I) synthesis, modification, and characterization of biobased polymers; (II) new processes for the preparation of biobased monomers from natural feedstock; (III) the application of biobased polymers in biomedicine and tissue engineering; (IV) the application of biobased polymers in multiresponsive materials; (V) the application of biobased polymers in nanotechnology and in advanced 3D printing; (VI) the durability of biobased polymers; (VII) the degradation of biobased polymers; and (VIII) the life-cycle assessment analysis (LCA) on biobased polymers. 


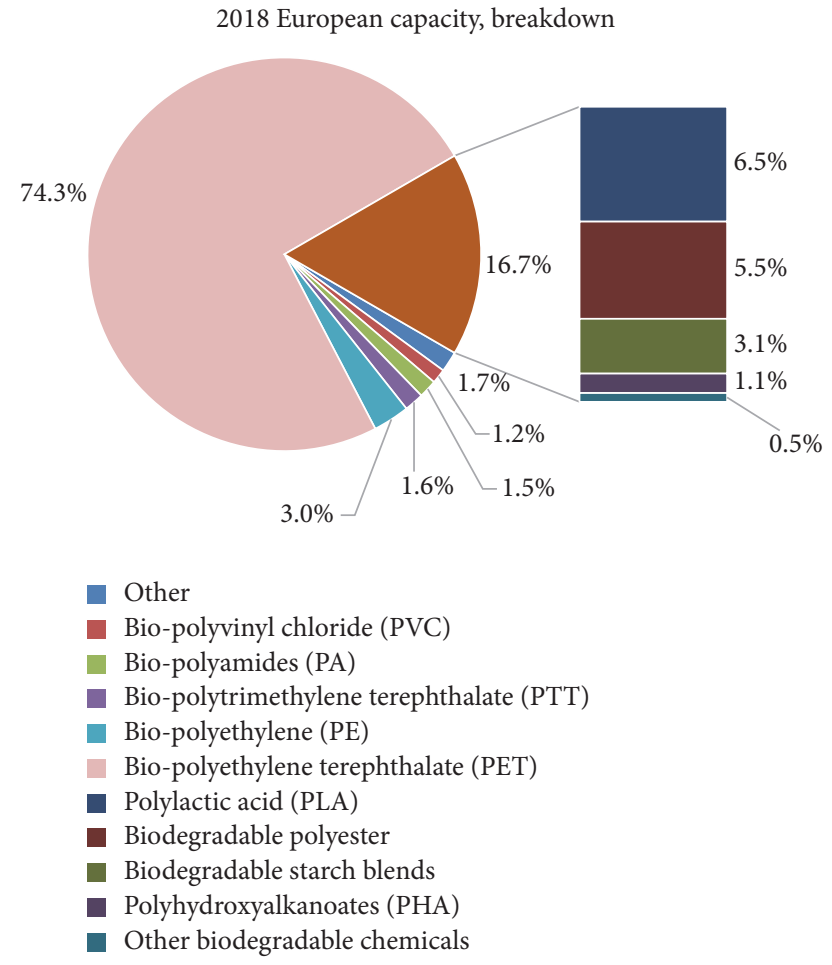

FIgURE 1: Bioplastics capacity: European breakdown.

We hope readers will benefit for their own research from this special issue.

\title{
Acknowledgments
}

We would like to express our appreciation to all the authors for their informative contributions and the reviewers for their support and constructive critiques in making this special issue possible.

\author{
Daniela Saviello \\ Daniele Cespi \\ Vinay Sharma \\ Shida Miao \\ Raffaele Cucciniello
}



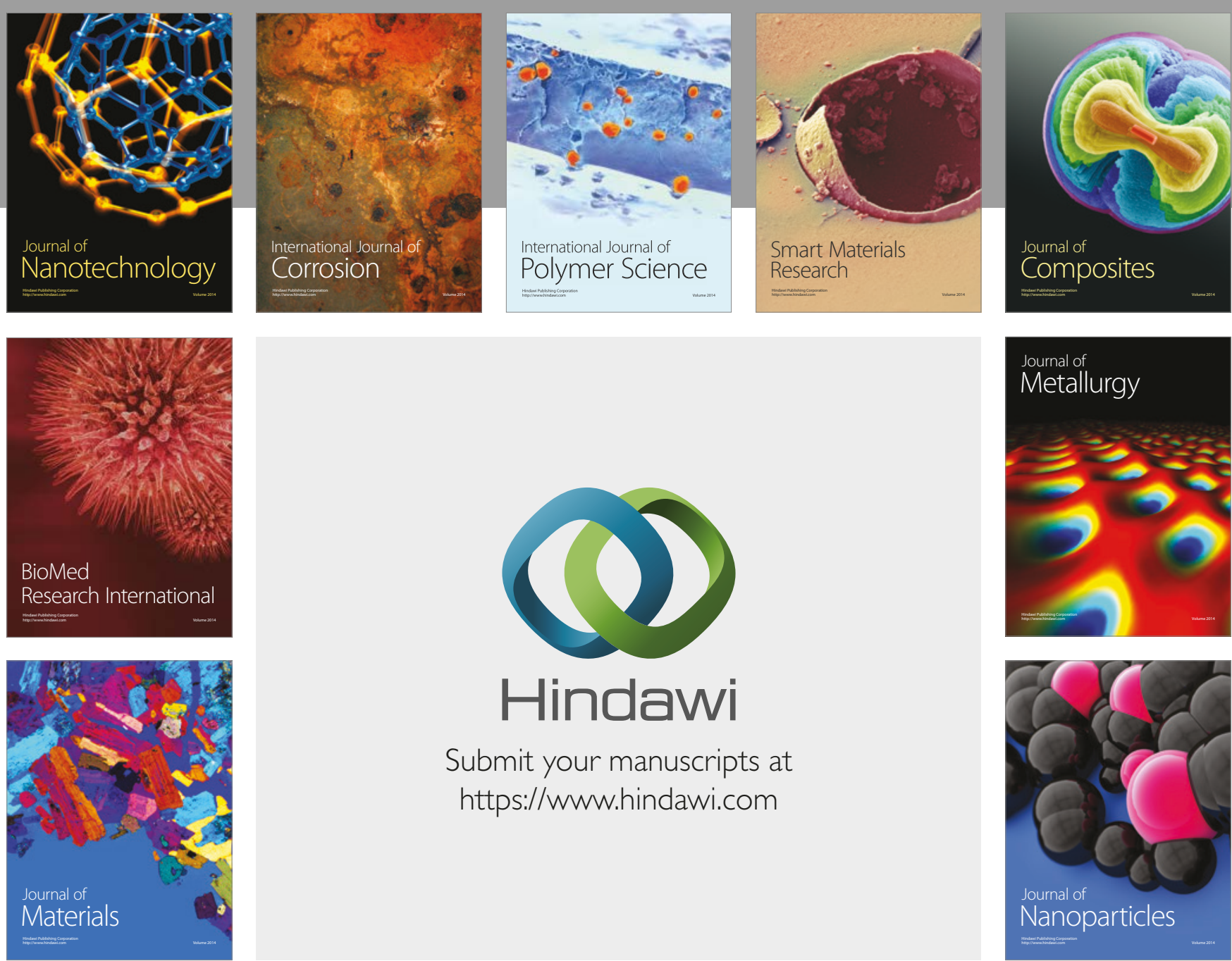

\section{Hindawi}

Submit your manuscripts at

https://www.hindawi.com
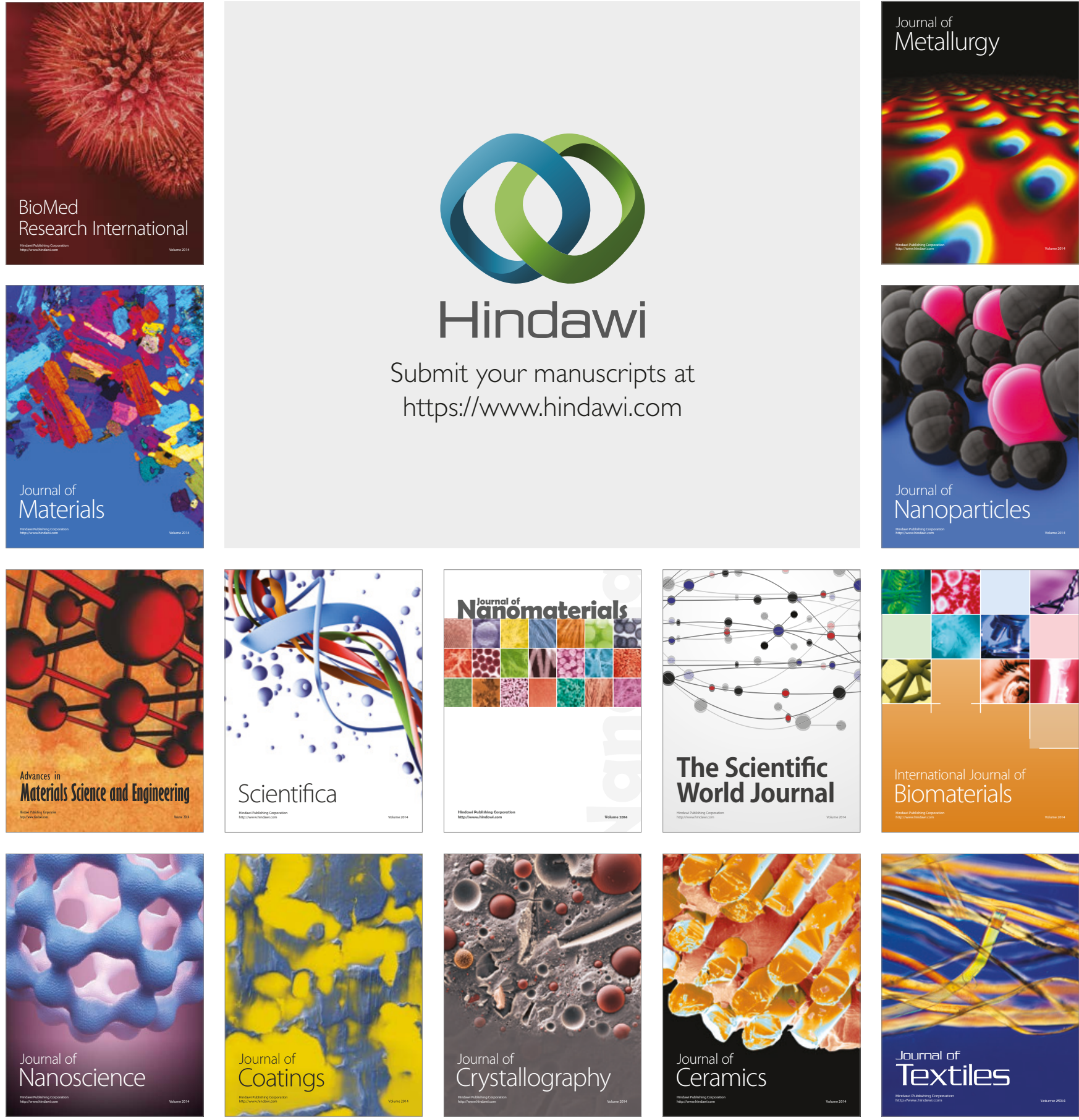

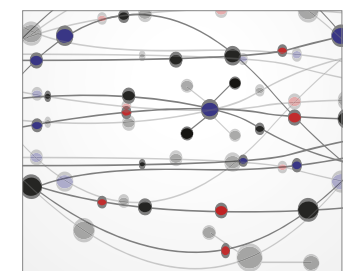

The Scientific World Journal
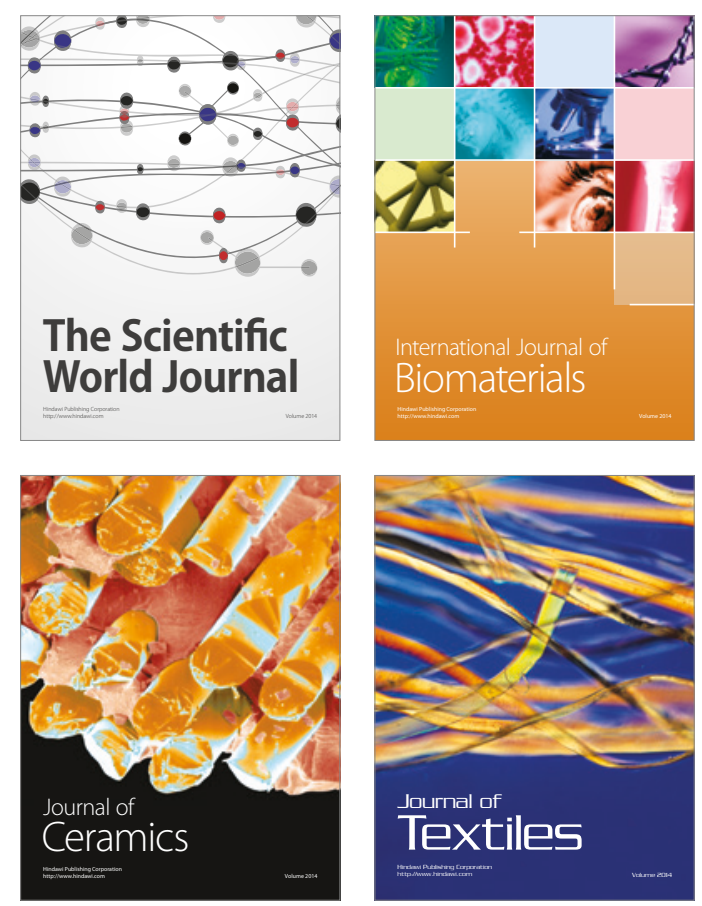